\title{
Computation of Carson formulas using piecewise approximation of kernel function
}

\author{
Ivan Krolo, Slavko Vujević, Tonći Modrić \\ Department of Electric Power Engineering, University of Split, Split, CROATIA \\ e-mail:ikrolo@fesb.hr
}

\section{SUMMARY}

Novel approach for the high-accurate computation of Carson formulas is presented. Carson formulas are used for computation of per-unit length (pul) self and mutual impedances of infinitely long parallel conductors. Numerical algorithm described in this paper uses a piecewise approximation of the kernel function which appears in the Carson formula corrections. Approximated kernel function is multiplied by the rest of the integrands in the impedance correction expressions and analytically integrated. By using the proposed algorithm, highaccurate results with the desired computed n-digit accuracy can easily be obtained. Results computed by the proposed algorithm are compared with the two most commonly used approximation methods for large frequency range.

KEY WORDS: Carson formulas; mutual impedance; numerical algorithm; piecewise approximation; self-impedance.

\section{INTRODUCTION}

The computation of pul self and mutual impedances of power line conductors is a well-known topic which first appeared at the beginning of the $20^{\text {th }}$ century. Exact formulas for the computation of pul self and mutual impedances of infinitely long parallel conductors were presented by Carson [1]. Carson formulas contain two integrals with infinite upper limits that cannot be analytically solved. Since then, many scientists and engineers have tried to solve this problem by using various approximation methods [1-8]. Carson integrals were developed for homogeneous earth containing the identical kernel function. This paper derives a new numerical algorithm that can successfully solve these two integrals by using piecewise approximation of the integrals' kernel function. In other words, kernel function is sampled, and sampled pieces are approximated by a line or parabola. Approximated kernel function is then multiplied by the rest of the integrands in the impedance correction expressions which enables analytical integration. Infinite upper limits of the Carson's integrals must be truncated in order to enable numerical solving $[9,10]$. The accuracy of the proposed algorithm depends on the total number of sample points and chosen truncated upper limits of the semi-infinite integrals. 
By increasing the total number of sample points, computed results reliably tend to exact values. In the event when the doubling and quadrupling of the total number of sampled points yields the same $n$-digit results, it can be concluded that the desired $n$-digit accuracy is achieved. Therefore, the proposed high-accurate algorithm can achieve the desired computed n-digit accurate results.

This paper provides a summarized account of two widespread approximation methods of the Carson formulas. The first is referred to as "single-term approximation method", proposed by Carson himself, where his infinite series were approximated by their first terms [1,11-13]. The second one was proposed by Dubanton [3], described by Gary [4] and mathematically verified by Deri [5], where they proposed the imagined depth of the earth return conductors. Numerical examples show the comparison of proposed algorithms and comparison of results obtained by the proposed algorithms and the two approximation methods.

\section{EXACT CARSON FORMULAS}

Formulas used for the computation of self and mutual impedances were proposed by Carson [1]. It is important to say that they neglect displacement currents. They were developed for infinitely long parallel conductors and homogeneous earth. Figure 1 shows two parallel cylindrical conductors in the air, their images and all relevant distances between them.

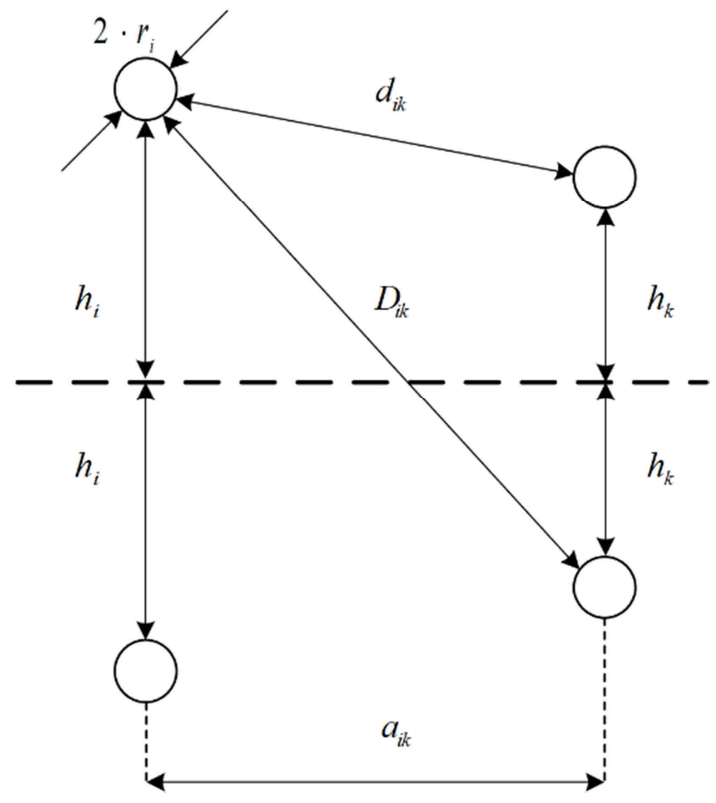

Fig. 1 Relevant distances between two conductors in the air and their images

Exact formulas for pul self and mutual impedances can be divided into two parts $[9,10]$ :

$$
\begin{aligned}
& \bar{Z}_{i i}^{1}=\bar{Z}_{i i}^{1 p c g}+\bar{Z}_{i i}^{1 c o r} \\
& \bar{Z}_{i k}^{1}=\bar{Z}_{i k}^{1 p c g}+\bar{Z}_{i k}^{1 c o r}
\end{aligned}
$$

where $\bar{Z}_{i i}^{1 p c g}$ and $\bar{Z}_{i k}^{1 p c g}$ are pul self and mutual impedances of perfectly conducting ground, whereas $\bar{Z}_{i i}^{1 c o r}$ and $\bar{Z}_{i k}^{1 c o r}$ are pul self and mutual impedance corrections. Pul self and mutual impedances of the perfectly conducting ground can be written as: 


$$
\begin{gathered}
\bar{Z}_{i i}^{1 p c g}=\bar{Z}_{i}^{1}+j \cdot \frac{\omega \cdot \mu_{0}}{2 \cdot \pi} \cdot \ln \frac{2 \cdot h_{i}}{r_{i}} \\
\bar{Z}_{i k}^{1 p c g}=j \cdot \frac{\omega \cdot \mu_{0}}{2 \cdot \pi} \cdot \ln \frac{D_{i k}}{d_{i k}}
\end{gathered}
$$

where $\bar{Z}_{i}^{1}$ is the pul internal impedance of conductor, $h_{i}$ is the $i^{\text {th }}$ conductor height, $D_{i k}$ is the distance between $i^{\text {th }}$ conductor and the $k^{\text {th }}$ conductor image, $d_{i k}$ is the distance between $i^{\text {th }}$ and $k$ th conductor, $r_{i}$ is the $i^{\text {th }}$ conductor radius, $\omega$ is the angular frequency and $\mu_{0}$ is the magnetic permeability of vacuum. Distances $D_{i k}$ and $d_{i k}$ are defined by:

$$
\begin{aligned}
& D_{i k}=\sqrt{a_{i k}^{2}+\left(h_{i}+h_{k}\right)^{2}} \\
& d_{i k}=\sqrt{a_{i k}^{2}+\left(h_{i}-h_{k}\right)^{2}}
\end{aligned}
$$

where $h_{k}$ is the $k^{\text {th }}$ conductor height and $a_{i k}$ is the horizontal distance between $i^{\text {th }}$ and $k^{\text {th }}$ conductors.

Exact formulas for pul self and mutual impedance corrections can be written as [1]:

$$
\begin{gathered}
\bar{Z}_{i i}^{1 \text { cor }}=\frac{\omega \cdot \mu_{0}}{\pi} \cdot \int_{0}^{\infty}\left(\sqrt{\lambda^{2}+j}-\lambda\right) \cdot e^{-p \cdot \lambda} \cdot d \lambda \\
\bar{Z}_{i k}^{1 c o r}=\frac{\omega \cdot \mu_{0}}{\pi} \cdot \int_{0}^{\infty}\left(\sqrt{\lambda^{2}+j}-\lambda\right) \cdot e^{-p \cdot \lambda} \cdot \cos (q \cdot \lambda) \cdot d \lambda
\end{gathered}
$$

where:

$$
\begin{gathered}
q=a_{i k} \cdot \gamma \\
p=\left\{\begin{array}{lll}
2 \cdot h_{i} \cdot \gamma & \text { for } \quad i=k \\
\left(h_{i}+h_{k}\right) \cdot \gamma & \text { for } \quad i \neq k
\end{array}\right.
\end{gathered}
$$

Complex wave propagation constant $\bar{\gamma}$ and its magnitude $\gamma$ are described by:

$$
\bar{\gamma}=\frac{\gamma}{\sqrt{2}} \cdot(1+j) ; \gamma=\sqrt{\frac{\omega \cdot \mu_{0}}{\rho}}
$$

where $\rho$ represents soil resistivity. Kernel function of the integrals in (7) and (8) is identical for both cases, and, most importantly, independent of soil characteristics and frequency. It can be divided into two parts $[9,10]$ :

$$
\bar{f}=\sqrt{\lambda^{2}+j}-\lambda=f^{r}+j \cdot f^{i}
$$

It must be emphasised that only the positive solution of the square root is considered, meaning that the solution is placed in the first quadrant [14]. The real and the imaginary part of the kernel function are (Figure 2):

$$
f^{r}=\frac{1}{(\sqrt{2} \cdot g+2 \cdot \lambda) \cdot g^{2}} \quad ; \quad f^{i}=\frac{1}{\sqrt{2} \cdot g}
$$

where:

$$
g=\sqrt{\lambda^{2}+\sqrt{\lambda^{4}+1}}
$$



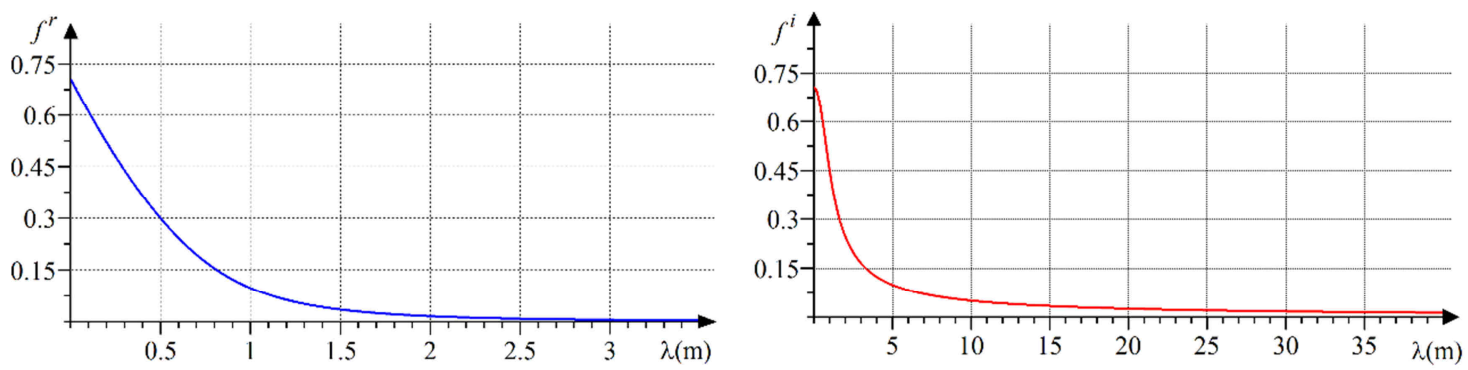

Fig. 2 Real and imaginary part of kernel function

\section{HIGH-ACCURATE NUMERICAL COMPUTATION OF CARSON FORMULAS}

\subsection{COMPUTATION OF PUL INTERNAL IMPEDANCE}

Pul internal impedance of conductor must be computed for pul self-impedance of the perfectly conducted ground. High-accurate computation of pul internal impedance of solid and tubular cylindrical single-layer and two-layer conductors (Figure 3), at power frequency, can be performed by using the scaled Bessel and Neumman functions [15]. This computation method is valid only for small complex arguments (low frequency, low permeability and small dimension of the conductor). For large complex arguments, improved method with scaled modified Bessel functions for single-layer [16] and two-layer [17] conductors must be used.

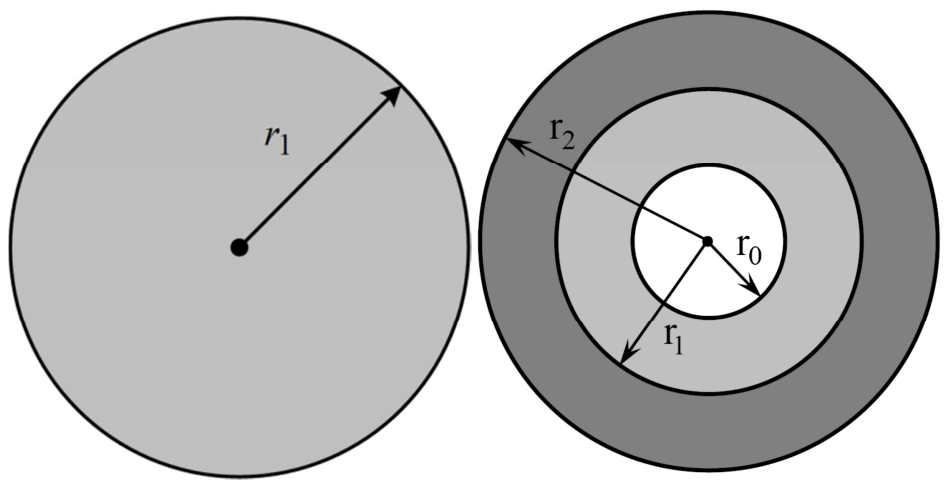

Fig. 3 Cross-section of a single-layer solid and two-layer tubular cylindrical conductors

\subsection{TRUNCATION OF SEMI-INFINITE INTEGRALS}

Numerical computations cannot handle infinite values, hence semi-infinite limits of integrals (7) and (8) must be truncated. Therefore, pul self and mutual impedance corrections can be written as:

$$
\begin{gathered}
\bar{Z}_{i i}^{1 c o r}=\frac{\omega \cdot \mu_{0}}{\pi} \cdot\left(\int_{0}^{\lambda_{r m}} f^{r} \cdot e^{-p \cdot \lambda} \cdot d \lambda+j \cdot \int_{0}^{\lambda_{i m}} f^{i} \cdot e^{-p \cdot \lambda} \cdot d \lambda\right) \\
\bar{Z}_{i k}^{1 c o r}=\frac{\omega \cdot \mu_{0}}{\pi} \cdot\left[\int_{0}^{\lambda_{r m}} f^{r} \cdot e^{-p \cdot \lambda} \cdot \cos (q \cdot \lambda) \cdot d \lambda+j \cdot \int_{0}^{\lambda_{i m}} f^{i} \cdot e^{-p \cdot \lambda} \cdot \cos (q \cdot \lambda) \cdot d \lambda\right]
\end{gathered}
$$


The finite upper limits, $\lambda_{r m}$ for the real part and $\lambda_{i m}$ for the imaginary part of the kernel function can be computed by using the asymptotic approximation of the kernel function for large arguments:

$$
\bar{f}_{a}(\lambda)=f_{a}^{r}+j \cdot f_{a}^{i}=\frac{1}{8 \cdot \lambda^{3}}+j \cdot \frac{1}{2 \cdot \lambda}
$$

By combining (17) with (15) and (16), the following nonlinear equations are obtained:

$$
\begin{aligned}
f_{a}^{r} \cdot e^{-p \cdot \lambda_{r m}} & =\frac{e^{-p \cdot \lambda_{r m}}}{8 \cdot \lambda_{r m}^{3}}=10^{m} \\
f_{a}^{i} \cdot e^{-p \cdot \lambda_{i m}} & =\frac{e^{-p \cdot \lambda_{i m}}}{2 \cdot \lambda_{i m}}=10^{m}
\end{aligned}
$$

where $m$ is the order of magnitude on which the accuracy of approximation depends. It is obvious that $\lambda_{i m}$ will always be higher than $\lambda_{r m}$ because the imaginary part of the kernel function has a much slower rate of decay toward zero, which can be clearly seen in Figure 2 . Nonlinear equations (20) and (21) can be solved by using the Newton-Raphson method [18].

\subsection{PIECEWISE LINEAR APPROXIMATION}

Piecewise linear algorithm approximates the kernel function linearly between two successive sample points (Figure 4). Piecewise linear approximations of the real and the imaginary part of the kernel function, for $\lambda_{k} \leq \lambda \leq \lambda_{k+1}$, are described by:

$$
\begin{gathered}
f^{r}=s_{k}^{r} \cdot \lambda+\ell_{k}^{r} ; \quad k=1,2, \ldots, N R-1 \\
f^{i}=s_{k}^{i} \cdot \lambda+\ell_{k}^{i} ; \quad k=1,2, \ldots, N I-1
\end{gathered}
$$

where $s_{k}^{r}, s_{k}^{i}$ are slopes and $l_{k}^{r}, l_{k}^{r}$ are $y$-intercepts of the linearly approximated kernel function. They are described by:

$$
\begin{aligned}
& s_{k}^{r}=\frac{f_{k+1}^{r}-f_{k}^{r}}{\lambda_{k+1}-\lambda_{k}} ; \quad s_{k}^{i}=\frac{f_{k+1}^{i}-f_{k}^{i}}{\lambda_{k+1}-\lambda_{k}} \\
& \ell_{k}^{r}=f_{k}^{r}-k_{k}^{r} \cdot \lambda_{k} ; \quad \ell_{k}^{i}=f_{k}^{i}-k_{k}^{i} \cdot \lambda_{k}
\end{aligned}
$$

where $N R$ and $N I$ are the total number of sample points for the real and the imaginary part of the kernel function, respectively.

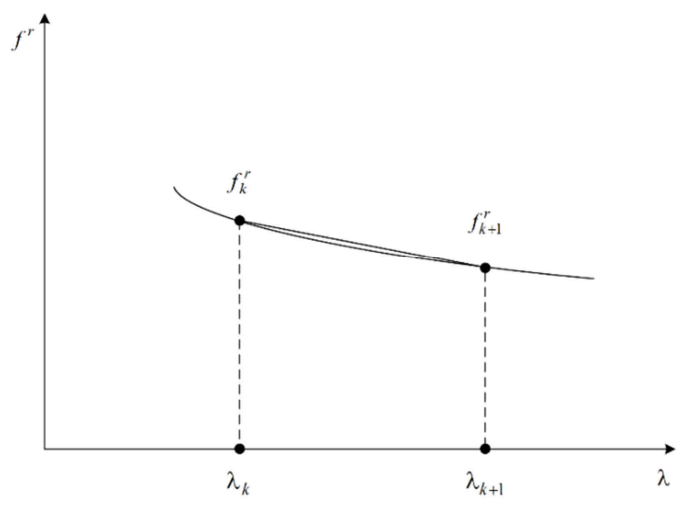

Fig. 4 Piecewise linear approximation of the real part of kernel function 
Expressions for impedance corrections (15) and (16) can now be written as:

$$
\begin{gathered}
\bar{Z}_{i i}^{1 c o r}=\frac{\omega \cdot \mu_{0}}{\pi} \cdot \sum_{k=1}^{N R-1}\left(s_{k}^{r} \cdot \int_{\lambda_{k}}^{\lambda_{k+1}} \lambda \cdot e^{-p \cdot \lambda} \cdot d \lambda+\ell_{k}^{r} \cdot \int_{\lambda_{k}}^{\lambda_{k+1}} e^{-p \cdot \lambda} \cdot d \lambda\right)+ \\
+\frac{j \cdot \omega \cdot \mu_{0}}{\pi} \cdot \sum_{k=1}^{N I-1}\left(s_{k}^{i} \cdot \int_{\lambda_{k}}^{\lambda_{k+1}} \lambda \cdot e^{-p \cdot \lambda} \cdot d \lambda+\ell_{k}^{i} \cdot \int_{\lambda_{k}}^{\lambda_{k+1}} e^{-p \cdot \lambda} \cdot d \lambda\right) \\
\bar{Z}_{i k}^{1 c o r}=\frac{\omega \cdot \mu_{0}}{\pi} \cdot \sum_{k=1}^{N R-1}\left[s_{k}^{r} \cdot \int_{\lambda_{k}}^{\lambda_{k+1}} \lambda \cdot e^{-p \cdot \lambda} \cdot \cos (q \cdot \lambda) \cdot d \lambda+\ell_{k}^{r} \cdot \int_{\lambda_{k}}^{\lambda_{k+1}} e^{-p \cdot \lambda} \cdot \cos (q \cdot \lambda) \cdot d \lambda\right]+ \\
+\frac{j \cdot \omega \cdot \mu_{0}}{\pi} \cdot \sum_{k=1}^{N I-1}\left[s_{k}^{i} \cdot \int_{\lambda_{k}}^{\lambda_{k+1}} \lambda \cdot e^{-p \cdot \lambda} \cdot \cos (q \cdot \lambda) \cdot d \lambda+\ell_{k}^{i} \cdot \int_{\lambda_{k}}^{\lambda_{k+1}} e^{-p \cdot \lambda} \cdot \cos (q \cdot \lambda) \cdot d \lambda\right]
\end{gathered}
$$

After analytical integration, (24) and (25) become:

$$
\begin{aligned}
\bar{Z}_{i i}^{1 c o r} & =\frac{\omega \cdot \mu_{0}}{\pi} \cdot \sum_{k=1}^{N R-1}\left\{s_{k}^{r} \cdot\left[I_{1}\left(\lambda_{k+1}\right)-I_{1}\left(\lambda_{k}\right)\right]+\ell_{k}^{r} \cdot\left[I_{2}\left(\lambda_{k+1}\right)-I_{2}\left(\lambda_{k}\right)\right]\right\}+ \\
& +\frac{j \cdot \omega \cdot \mu_{0}}{\pi} \cdot \sum_{k=1}^{N I-1}\left\{s_{k}^{i} \cdot\left[I_{1}\left(\lambda_{k+1}\right)-I_{1}\left(\lambda_{k}\right)\right]+\ell_{k}^{i} \cdot\left[I_{2}\left(\lambda_{k+1}\right)-I_{2}\left(\lambda_{k}\right)\right]\right\} \\
\bar{Z}_{i k}^{1 c o r} & =\frac{\omega \cdot \mu_{0}}{\pi} \cdot \sum_{k=1}^{N R-1}\left\{s_{k}^{r} \cdot\left[I_{3}\left(\lambda_{k+1}\right)-I_{3}\left(\lambda_{k}\right)\right]+\ell_{k}^{r} \cdot\left[I_{4}\left(\lambda_{k+1}\right)-I_{4}\left(\lambda_{k}\right)\right]\right\}+ \\
& +\frac{j \cdot \omega \cdot \mu_{0}}{\pi} \cdot \sum_{k=1}^{N I-1}\left\{s_{k}^{i} \cdot\left[I_{3}\left(\lambda_{k+1}\right)-I_{3}\left(\lambda_{k}\right)\right]+\ell_{k}^{i} \cdot\left[I_{4}\left(\lambda_{k+1}\right)-I_{4}\left(\lambda_{k}\right)\right]\right\}
\end{aligned}
$$

where:

$$
\begin{gathered}
I_{1}(\lambda)=\int \lambda \cdot e^{-p \cdot \lambda} \cdot d \lambda=-\frac{e^{-p \cdot \lambda}}{p^{2}} \cdot(p \cdot \lambda+1) \\
I_{2}(\lambda)=\int e^{-p \cdot \lambda} \cdot d \lambda=-\frac{e^{-p \cdot \lambda}}{p} \\
I_{3}(\lambda)=\int \lambda \cdot e^{-p \cdot \lambda} \cdot \cos (q \cdot \lambda) \cdot d \lambda=\frac{\lambda \cdot e^{-p \cdot \lambda}}{p^{2}+q^{2}} \cdot[q \cdot \sin (q \cdot \lambda)-p \cdot \cos (q \cdot \lambda)]- \\
-\frac{e^{-p \cdot \lambda}}{\left(p^{2}+q^{2}\right)^{2}} \cdot\left[\left(p^{2}-q^{2}\right) \cdot \cos (q \cdot \lambda)-2 \cdot p \cdot q \cdot \sin (q \cdot \lambda)\right] \\
I_{4}(\lambda)=\int e^{-p \cdot \lambda} \cdot \cos (q \cdot \lambda) \cdot d \lambda=\frac{e^{-p \cdot \lambda}}{p^{2}+q^{2}} \cdot[q \cdot \sin (q \cdot \lambda)-p \cdot \cos (q \cdot \lambda)]
\end{gathered}
$$

Piecewise linear approximation algorithm can rather efficiently approximate the kernel function because the sampling of the kernel function can be performed until the desired computed $n$-digit accuracy is achieved. Approximation accuracy is a key factor that defines accuracy of the overall algorithm because the approximated kernel function is multiplied by 
the rest of the integrands in the impedance correction expressions and then analytically integrated.

\subsection{PIECEWISE QUADRATIC APPROXIMATION}

Piecewise quadratic approximation algorithm can be considered as an improved piecewise linear approximation algorithm. This algorithm has a slightly more complicated expressions, although it demands less sampling points for the same accuracy degree of results. This is a more appropriate algorithm for robust computations since it is less time-consuming than the piecewise linear algorithm. Piecewise quadratic algorithm approximates the kernel function by a parabola. Parabola of the sampled finite element is defined by three points (Figure 5).

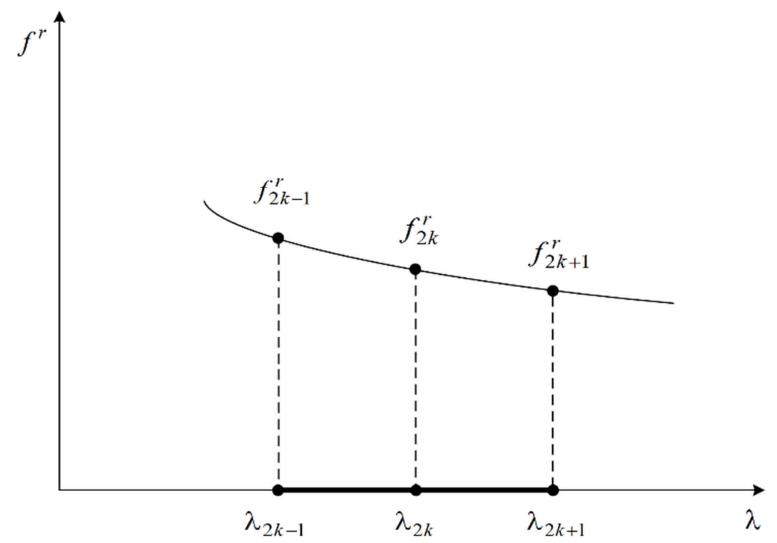

Fig. 5 Sample points of the kth finite element for the real part of the kernel function

For the $k$ th finite element in interval $\lambda_{2 k-1} \leq \lambda \leq \lambda_{2 k+1}$, the real and the imaginary part of the kernel function are described as follows:

$$
\begin{aligned}
f^{r} & =a_{k}^{r} \cdot \lambda^{2}+b_{k}^{r} \cdot \lambda+c_{k}^{r} ; \quad k=1,2, \ldots, N E R \\
f^{i} & =a_{k}^{i} \cdot \lambda^{2}+b_{k}^{i} \cdot \lambda+c_{k}^{i} ; \quad k=1,2, \ldots, N E I
\end{aligned}
$$

where NER and NEI are the total number of finite elements for the real and the imaginary part of the kernel function, respectively. The coefficients of the parabolas are:

$$
\begin{aligned}
a_{k}^{r} & =\frac{2}{\left(\lambda_{2 k+1}-\lambda_{2 k-1}\right)^{2}} \cdot\left(f_{2 k-1}^{r}-2 \cdot f_{2 k}^{r}+f_{2 k+1}^{r}\right) \\
a_{k}^{i} & =\frac{2}{\left(\lambda_{2 k+1}-\lambda_{2 k-1}\right)^{2}} \cdot\left(f_{2 k-1}^{i}-2 \cdot f_{2 k}^{i}+f_{2 k+1}^{i}\right) \\
b_{k}^{r} & =\frac{2}{\left(\lambda_{2 k+1}-\lambda_{2 k-1}\right)^{2}} \cdot\left[2 \cdot\left(\lambda_{2 k-1}+\lambda_{2 k+1}\right) \cdot f_{2 k}^{r}-\right. \\
& \left.-\left(\lambda_{2 k}+\lambda_{2 k+1}\right) \cdot f_{2 k-1}^{r}-\left(\lambda_{2 k-1}+\lambda_{2 k}\right) \cdot f_{2 k+1}^{r}\right] \\
b_{k}^{i} & =\frac{2}{\left(\lambda_{2 k+1}-\lambda_{2 k-1}\right)^{2}} \cdot\left[2 \cdot\left(\lambda_{2 k-1}+\lambda_{2 k+1}\right) \cdot f_{2 k}^{i}-\right. \\
& \left.-\left(\lambda_{2 k}+\lambda_{2 k+1}\right) \cdot f_{2 k-1}^{i}-\left(\lambda_{2 k-1}+\lambda_{2 k}\right) \cdot f_{2 k+1}^{i}\right]
\end{aligned}
$$




$$
\begin{aligned}
c_{k}^{r} & =\frac{2}{\left(\lambda_{2 k+1}-\lambda_{2 k-1}\right)^{2}} \cdot\left[\lambda_{2 k} \cdot \lambda_{2 k+1} \cdot f_{2 k-1}^{r}-\right. \\
& \left.-2 \cdot \lambda_{2 k-1} \cdot \lambda_{2 k+1} \cdot f_{2 k}^{r}+\lambda_{2 k-1} \cdot \lambda_{2 k} \cdot f_{2 k+1}^{r}\right] \\
c_{k}^{i} & =\frac{2}{\left(\lambda_{2 k+1}-\lambda_{2 k-1}\right)^{2}} \cdot\left[\lambda_{2 k} \cdot \lambda_{2 k+1} \cdot f_{2 k-1}^{i}-\right. \\
& \left.-2 \cdot \lambda_{2 k-1} \cdot \lambda_{2 k+1} \cdot f_{2 k}^{i}+\lambda_{2 k-1} \cdot \lambda_{2 k} \cdot f_{2 k+1}^{i}\right]
\end{aligned}
$$

Equations (34)-(36) are derived with the assumption that $\lambda_{2 k}$ is the mid-point of the $k$ th finite element:

$$
\lambda_{2 k}=\frac{\lambda_{2 k+1}+\lambda_{2 k-1}}{2}
$$

This means that the odd points of each finite element are sampled arbitrarily, depending on the demanded accuracy of the result, whereas the abscissas of the even points are computed by (37). The total number of sample points for the real and the imaginary part of the kernel function are:

$$
N R=2 \cdot N E R+1 ; \quad N I=2 \cdot N E I+1
$$

respectively.

As in the piecewise linear algorithm, the approximated kernel function is multiplied by the rest of the integrands. Thus (15) and (16) are now written as:

$$
\begin{aligned}
\bar{Z}_{i i}^{1 c o r} & =\frac{\omega \cdot \mu_{0}}{\pi} \cdot \sum_{k=1}^{N E R}\left(a_{k}^{r} \cdot \int_{\lambda_{2 k-1}}^{\lambda_{2 k+1}} \lambda^{2} \cdot e^{-p \cdot \lambda} \cdot d \lambda+b_{k}^{r} \cdot \int_{\lambda_{2 k-1}}^{\lambda_{2 k+1}} \lambda \cdot e^{-p \cdot \lambda} \cdot d \lambda+c_{k}^{r} \cdot \int_{\lambda_{2 k-1}}^{\lambda_{2 k+1}} e^{-p \cdot \lambda} \cdot d \lambda\right)+ \\
& +\frac{j \cdot \omega \cdot \mu_{0}}{\pi} \cdot \sum_{k=1}^{N E I}\left(a_{k}^{i} \cdot \int_{\lambda_{2 k-1}}^{\lambda_{2 k+1}} \lambda^{2} \cdot e^{-p \cdot \lambda} \cdot d \lambda+b_{k}^{i} \cdot \int_{\lambda_{2 k-1}}^{\lambda_{2 k+1}} \lambda \cdot e^{-p \cdot \lambda} \cdot d \lambda+c_{k}^{i} \cdot \int_{\lambda_{2 k-1}}^{\lambda_{2 k+1}} e^{-p \cdot \lambda} \cdot d \lambda\right) \\
\bar{Z}_{i k}^{1 c o r} & =\frac{\omega \cdot \mu_{0}}{\pi} \cdot \sum_{k=1}^{N E R}\left[a_{k}^{r} \cdot \int_{\lambda_{2 k-1}}^{\lambda_{2 k+1}} \lambda^{2} \cdot e^{-p \cdot \lambda} \cdot \cos (q \cdot \lambda) \cdot d \lambda+b_{k}^{r} \cdot \int_{\lambda_{2 k-1}}^{\lambda_{2 k+1}} \lambda \cdot e^{-p \cdot \lambda} \cdot \cos (q \cdot \lambda) \cdot d \lambda+\right. \\
& \left.+c_{k}^{r} \cdot \int_{\lambda_{2 k-1}}^{r k+1} e^{-p \cdot \lambda} \cdot \cos (q \cdot \lambda) \cdot d \lambda\right]+\frac{j \cdot \omega \cdot \mu_{0}}{\pi} \cdot \sum_{k=1}^{N E I}\left[a_{k}^{i} \cdot \int_{\lambda_{2 k-1}}^{\lambda_{2 k+1}} \lambda^{2} \cdot e^{-p \cdot \lambda} \cdot \cos (q \cdot \lambda) \cdot d \lambda+\right. \\
& \left.+b_{k}^{i} \cdot \int_{\lambda_{2 k-1}}^{\lambda_{2 k+1}} \lambda \cdot e^{-p \cdot \lambda} \cdot \cos (q \cdot \lambda) \cdot d \lambda+c_{k}^{i} \cdot \int_{\lambda_{2 k-1}}^{\lambda_{2 k+1}} e^{-p \cdot \lambda} \cdot \cos (q \cdot \lambda) \cdot d \lambda\right]
\end{aligned}
$$

After analytical integration, (39) and (40) become:

$$
\begin{aligned}
\bar{Z}_{i i}^{1 c o r} & =\frac{\omega \cdot \mu_{0}}{\pi} \cdot \sum_{k=1}^{N E R}\left\{a_{k}^{r} \cdot\left[I_{5}\left(\lambda_{2 k+1}\right)-I_{5}\left(\lambda_{2 k-1}\right)\right]+b_{k}^{r} \cdot\left[I_{1}\left(\lambda_{2 k+1}\right)-I_{1}\left(\lambda_{2 k-1}\right)\right]+\right. \\
& \left.+c_{k}^{r} \cdot\left[I_{2}\left(\lambda_{2 k+1}\right)-I_{2}\left(\lambda_{2 k-1}\right)\right]\right\}+\frac{j \cdot \omega \cdot \mu_{0}}{\pi} \cdot \sum_{k=1}^{N E I}\left\{a_{k}^{i} \cdot\left[I_{5}\left(\lambda_{2 k+1}\right)-I_{5}\left(\lambda_{2 k-1}\right)\right]+\right. \\
& \left.+b_{k}^{i} \cdot\left[I_{1}\left(\lambda_{2 k+1}\right)-I_{1}\left(\lambda_{2 k-1}\right)\right]+c_{k}^{i} \cdot\left[I_{2}\left(\lambda_{2 k+1}\right)-I_{2}\left(\lambda_{2 k-1}\right)\right]\right\}
\end{aligned}
$$




$$
\begin{aligned}
\bar{Z}_{i k}^{1 c o r} & =\frac{\omega \cdot \mu_{0}}{\pi} \cdot \sum_{k=1}^{N E R}\left\{a_{k}^{r} \cdot\left[I_{6}\left(\lambda_{2 k+1}\right)-I_{6}\left(\lambda_{2 k-1}\right)\right]+b_{k}^{r} \cdot\left[I_{3}\left(\lambda_{2 k+1}\right)-I_{3}\left(\lambda_{2 k-1}\right)\right]+\right. \\
& \left.+c_{k}^{r} \cdot\left[I_{4}\left(\lambda_{2 k+1}\right)-I_{4}\left(\lambda_{2 k-1}\right)\right]\right\}+\frac{j \cdot \omega \cdot \mu_{0}}{\pi} \cdot \sum_{k=1}^{N E I}\left\{a_{k}^{i} \cdot\left[I_{6}\left(\lambda_{2 k+1}\right)-I_{6}\left(\lambda_{2 k-1}\right)\right]+\right. \\
& \left.+b_{k}^{i} \cdot\left[I_{3}\left(\lambda_{2 k+1}\right)-I_{3}\left(\lambda_{2 k-1}\right)\right]+c_{k}^{i} \cdot\left[I_{4}\left(\lambda_{2 k+1}\right)-I_{4}\left(\lambda_{2 k-1}\right)\right]\right\}
\end{aligned}
$$

where the solutions of integrals $I_{i}(\lambda) ; i \in\{1,4\}$ are given in (28)-(31), whereas:

$$
\begin{gathered}
I_{5}(\lambda)=\int \lambda^{2} \cdot e^{-p \cdot \lambda} \cdot d \lambda=-e^{-p \cdot \lambda} \cdot\left(\frac{\lambda^{2}}{p}+\frac{2 \cdot \lambda}{p^{2}}+\frac{2}{p^{3}}\right) \\
I_{6}(\lambda)=\int \lambda^{2} \cdot e^{-p \cdot \lambda} \cdot \cos (q \cdot \lambda) \cdot d \lambda= \\
=\frac{\lambda^{2} \cdot e^{-p \cdot \lambda}}{p^{2}+q^{2}} \cdot[q \cdot \sin (q \cdot \lambda)-p \cdot \cos (q \cdot \lambda)]+\frac{2 \cdot \lambda \cdot e^{-p \cdot \lambda}}{\left(p^{2}+q^{2}\right)^{2}} \cdot\left[2 \cdot p \cdot q \cdot \sin (q \cdot \lambda)-\left(p^{2}-q^{2}\right) \cdot \cos (q \cdot \lambda)\right]+ \\
+\frac{2 \cdot e^{-p \cdot \lambda}}{\left(p^{2}+q^{2}\right)^{3}} \cdot\left(3 \cdot p^{2} \cdot q-q^{3}\right) \cdot \sin (q \cdot \lambda)-\frac{2 \cdot e^{-p \cdot \lambda}}{\left(p^{2}+q^{2}\right)^{3}} \cdot\left(p^{3}-3 \cdot p \cdot q^{2}\right) \cdot \cos (q \cdot \lambda)
\end{gathered}
$$

Again, the sampling can be performed until the desired computed n-digit accuracy is achieved. Both numerical algorithms, piecewise linear and piecewise quadratic approximation algorithm, can easily be achieved by using the appropriate mathematical software.

\section{APPROXIMATE FORMULAS}

\subsection{SINGLE-TERM APPROXIMATION METHOD}

During the years, the exact Carson formulas for pul impedance corrections (7) and (8) obtained many approximations. The most commonly used approximation is the Single-term approximation, where two infinite Carson series for pul self and mutual impedance corrections are approximated by their first term. These approximate formulas were proposed by Carson himself [1], and they can be written as [11-13]:

$$
\begin{aligned}
& \bar{Z}_{i i}^{1 c o r}=\frac{\omega \cdot \mu_{0}}{8}+j \frac{\omega \cdot \mu_{0}}{2 \cdot \pi} \cdot \ln \frac{D_{e}}{2 \cdot h_{i}} \\
& \bar{Z}_{i k}^{1 c o r}=\frac{\omega \cdot \mu_{0}}{8}+j \frac{\omega \cdot \mu_{0}}{2 \cdot \pi} \cdot \ln \frac{D_{e}}{D_{i k}}
\end{aligned}
$$

where:

$$
D_{e}=658.8716 \cdot \sqrt{\frac{\rho}{f}}
$$

Formulas for pul self and mutual impedances, obtained by the sum of (3) and (4) with (45) and (46), thus become:

$$
\bar{Z}_{i i}^{1}=\bar{Z}_{i i}^{1 p c g}+\bar{Z}_{i i}^{1 c o r}=\bar{Z}_{i}^{1}+\frac{\omega \cdot \mu_{0}}{8}+j \cdot \frac{\omega \cdot \mu_{0}}{2 \cdot \pi} \cdot \ln \frac{D_{e}}{r_{i}}
$$




$$
\bar{Z}_{i k}^{1}=\bar{Z}_{i k}^{1 p c g}+\bar{Z}_{i k}^{1 c o r}=\frac{\omega \cdot \mu_{0}}{8}+j \cdot \frac{\omega \cdot \mu_{0}}{2 \cdot \pi} \cdot \ln \frac{D_{e}}{d_{i k}}
$$

It can be seen that (49) considers only the height difference between the conductors and not the actual height of the conductors above the ground. Hence, this is a rather rough approximation method, accurate only at low frequencies.

\subsection{GARY-DUBANTON FORMULAS}

Since the Single-term approximation method yielded poor results at higher frequencies, the researchers and engineers tried to develop a new method for the computation of Carson formulas. The new method was proposed by Dubanton [3], described by Gary [4], and mathematically described by Deri [5]. Pul self and mutual impedance corrections in this method are described by [5, 6]:

$$
\begin{gathered}
\bar{Z}_{i i}^{1 c o r}=j \frac{\omega \cdot \mu_{0}}{2 \cdot \pi} \cdot \ln \frac{h_{i}+\bar{\delta}}{h_{i}}=j \frac{\omega \cdot \mu_{0}}{2 \cdot \pi} \cdot \ln \frac{1+\bar{\gamma} \cdot h_{i}}{\bar{\gamma} \cdot h_{i}} \\
\bar{Z}_{i k}^{1 c o r}=j \frac{\omega \cdot \mu_{0}}{2 \cdot \pi} \cdot \ln \frac{\sqrt{\left(h_{i}+h_{k}+2 \cdot \bar{\delta}\right)^{2}+d_{i k}^{2}}}{D_{i k}}
\end{gathered}
$$

where $\bar{\delta}$ is the complex depth of earth return plane:

$$
\bar{\delta}=\frac{1}{\bar{\gamma}}=\frac{1-j}{\sqrt{2} \cdot \gamma}
$$

Complete formulas for pul self and mutual impedances, obtained by the sum of (3) and (4) with (50) and (51), are:

$$
\begin{gathered}
\bar{Z}_{i i}^{1}=\bar{Z}_{i i}^{1 p c g}+\bar{Z}_{i i}^{1 c o r}=\bar{Z}_{i}^{1}+j \cdot \frac{\omega \cdot \mu_{0}}{2 \cdot \pi} \cdot \ln \frac{2 \cdot\left(h_{i}+\bar{\delta}\right)}{r_{i}} \\
\bar{Z}_{i k}^{1}=\bar{Z}_{i k}^{1 p c g}+\bar{Z}_{i k}^{1 c o r}=j \frac{\omega \cdot \mu_{0}}{2 \cdot \pi} \cdot \ln \frac{\sqrt{\left(h_{i}+h_{k}+2 \cdot \bar{\delta}\right)^{2}+d_{i k}^{2}}}{d_{i k}}
\end{gathered}
$$

Contrary to (49), the approximate formula for pul mutual impedance (54) considers actual conductor heights. Therefore, this method is a more precise method, valid also at high frequencies.

\section{NUMERICAL EXAMPLES}

The numerical examples in this section show the computations of Carson formulas by using the above explained proposed algorithms and approximate methods. The computations were performed for cylindrical conductors characterised by the following data:

- $r_{i}=6.18 \mathrm{~mm}$

- $\sigma=58 \cdot 10^{6} \mathrm{~S} / \mathrm{m}$

- $\mu_{r}=1$

The geometry of the conductors is (Figure 1): 
- $h_{i}=10 \mathrm{~m} ; h_{k}=11 \mathrm{~m}$

- $a_{i k}=1 \mathrm{~m}$

\subsection{COMPARISON OF RESULTS COMPUTED BY PIECEWISE LINEAR AND PIECEWISE QUADRATIC APPROXIMATION OF THE KERNEL FUNCTION}

As explained in Section 3, the kernel function can be approximated by a line or parabola until the desired computed n-digit accuracy is obtained. This numerical example selected the 7-digit accurate result of the kernel function. Kernel functions' parts were divided into two intervals. For $0 \leq \lambda<1$ of the real and the imaginary part of the kernel function, the sample points were chosen to be equidistant in the linear scale, and for $\lambda \geq 1$, the sample points are chosen to be equidistant in the logarithmic scale. The truncated upper limits $\lambda_{r m}$ and $\lambda_{i m}$ depend on the order of magnitude $m$. In this numerical example, $m$ is equal to -10 . Further decreasing $m$ does not affect the selected 7-digit accuracy. Computations of pul self and mutual impedance corrections were performed for soil resistivity $\rho=500 \Omega \mathrm{m}$ and for power frequency $f=50 \mathrm{~Hz}$. The numbers of sample points required for both proposed algorithms are shown in Table 1.

Table 1 Numbers of sample points required for the identical 7-digit accurate result, obtained by piecewise linear and piecewise quadratic approximation algorithm

\begin{tabular}{|c|c|c|c|c|c|c|c|c|}
\hline \multirow{2}{*}{$\begin{array}{c}\text { Number of } \\
\text { sample } \\
\text { points }\end{array}$} & \multicolumn{4}{|c|}{ Piecewise linear approximation } & \multicolumn{4}{|c|}{ Piecewise quadratic approximation } \\
\hline & $\begin{array}{c}R e \\
\left(\bar{Z}_{i i}^{1 c o r}\right)\end{array}$ & $\left(\bar{Z}_{i i}^{1 \mathrm{Im}}\right)$ & $\begin{array}{c}R e \\
\left(\bar{Z}_{i k}^{1 c o r}\right)\end{array}$ & $\begin{array}{c}\operatorname{Im} \\
\left(\bar{Z}_{i k}^{1 \mathrm{cor}}\right)\end{array}$ & $\begin{array}{c}R e \\
\left(\bar{Z}_{i i}^{1 c o r}\right)\end{array}$ & $\left(\bar{Z}_{i i}^{1 \mathrm{Im}}\right)$ & $\begin{array}{c}R e \\
\left(\bar{Z}_{i k}^{1 c o r}\right)\end{array}$ & $\begin{array}{c}\operatorname{Im} \\
\left(\bar{Z}_{i k}^{1 c o r}\right)\end{array}$ \\
\hline $0 \leq \lambda<1$ & 1266 & 1266 & 1031 & 1031 & 23 & 23 & 24 & 24 \\
\hline$\lambda \geq 1$ & 11513 & 14147 & 11305 & 13871 & 178 & 218 & 169 & 207 \\
\hline $\begin{array}{c}\text { Total } \\
(N R / N I)\end{array}$ & 12779 & 15413 & 12336 & 14902 & 201 & 241 & 193 & 231 \\
\hline
\end{tabular}

The results in Table 1 show that the total number of sample points used by the piecewise quadratic approximation is significantly decreased in comparison with the piecewise linear approximation algorithm. The numerical tests show that the increase in soil resistivity significantly increases the total number of sample points. This makes the piecewise linear approximation algorithm inappropriate for computations with higher soil resistivities, whereas the piecewise quadratic algorithm can easily handle any soil resistivity.

\subsection{COMPUTATION OF PUL IMPEDANCE CORRECTIONS BY USING PIECEWISE QUADRATIC ALGORITHM AND APPROXIMATE FORMULAS}

As explained above, the piecewise linear and piecewise quadratic algorithms can obtain the identical results of pul self and mutual impedances by using different approximations of the kernel function.

This section depicts the computation of the real and imaginary parts of pul self and mutual impedance corrections of the cylindrical conductors. Computations were performed by using the proposed algorithms and approximate formulas for the frequency range $25 \mathrm{~Hz} \leq f \leq 10$ MHz. All computations were performed for three different homogeneous soil resistivities: 
- $\rho=10 \Omega m$

- $\rho=1000 \Omega m$

- $\rho=10000 \Omega m$

where $10 \Omega \mathrm{m}$ is considered to be the lowest, and $10000 \Omega \mathrm{m}$ the highest soil resistivity which may occur in measurements. Computed real and imaginary parts of pul impedance corrections are presented in the semi-logarithmic scale (Figure 6).
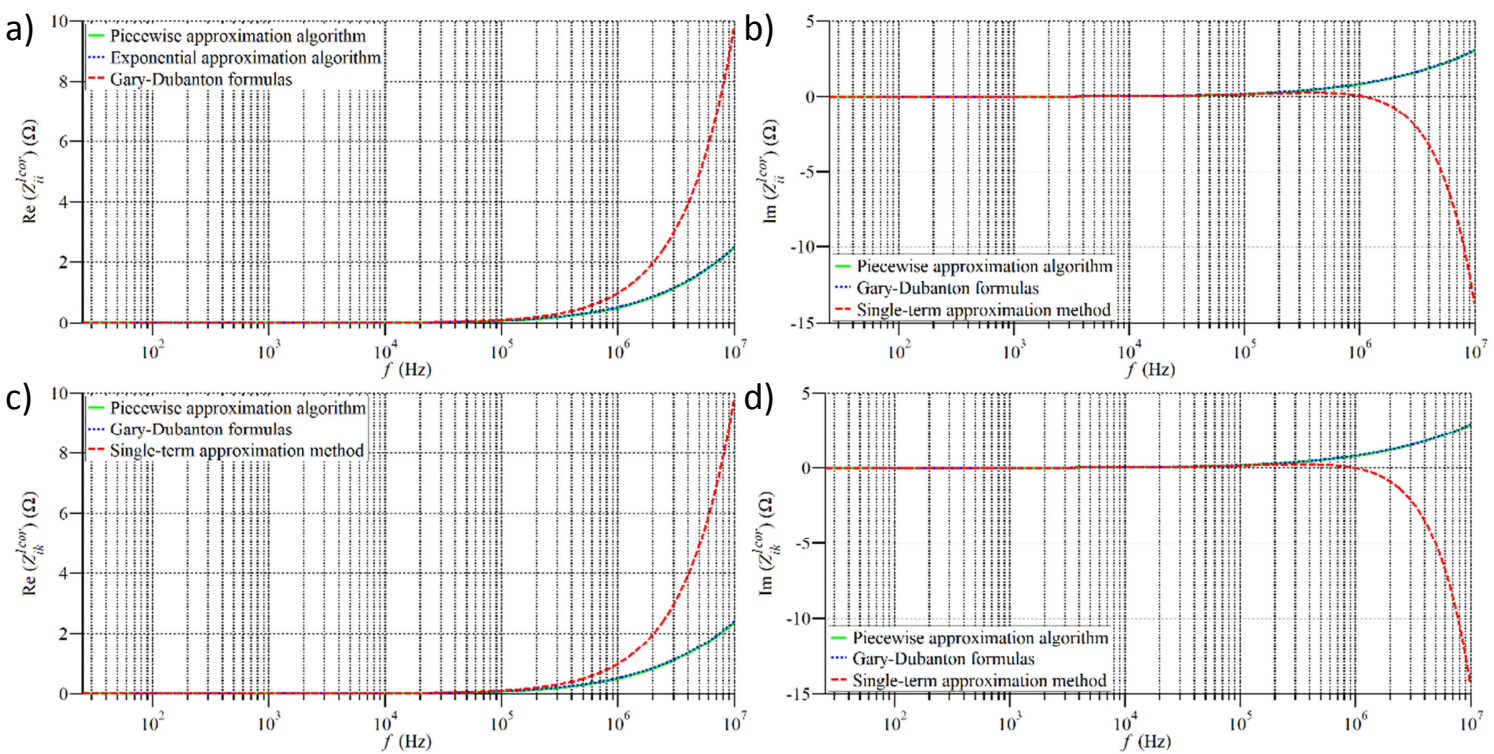

Fig. 6 Results obtained by piecewise approximation algorithm and approximate formulas for $\left.\left.\rho=1000 \Omega m: a) \operatorname{Re}\left(\bar{Z}_{i i}^{1 c o r}\right), b\right) \operatorname{Im}\left(\bar{Z}_{i i}^{1 c o r}\right), c\right) \operatorname{Re}\left(\bar{Z}_{i k}^{1 c o r}\right)$, and d) $\operatorname{Im}\left(\bar{Z}_{i k}^{1 c o r}\right)$

Results in Figure 6 show that the 7-digit accurate results obtained by the proposed algorithm and Gary-Dubanton formulas have almost identical curves through the large frequency range and in all cases. This verifies the Gary-Dubanton formulas as a reliable approximation method for the large frequency range. Figure 6 clearly demonstrates that the Single-term approximation method is valid only at low frequencies. In addition, the imaginary parts of both impedance corrections have negative values at high frequencies. The computations performed by using two other soil resistivities show a similar behaviour of the results for both pul impedance corrections, and therefore they were not graphically presented. Gary-Dubanton formulas obtain an almost identical curve as the proposed algorithm in all cases, hence Figures 7 to 9 show the percent errors of Gary-Dubanton formulas in relation to the 7-digit accurate results obtained by the proposed algorithm, for all considered cases.
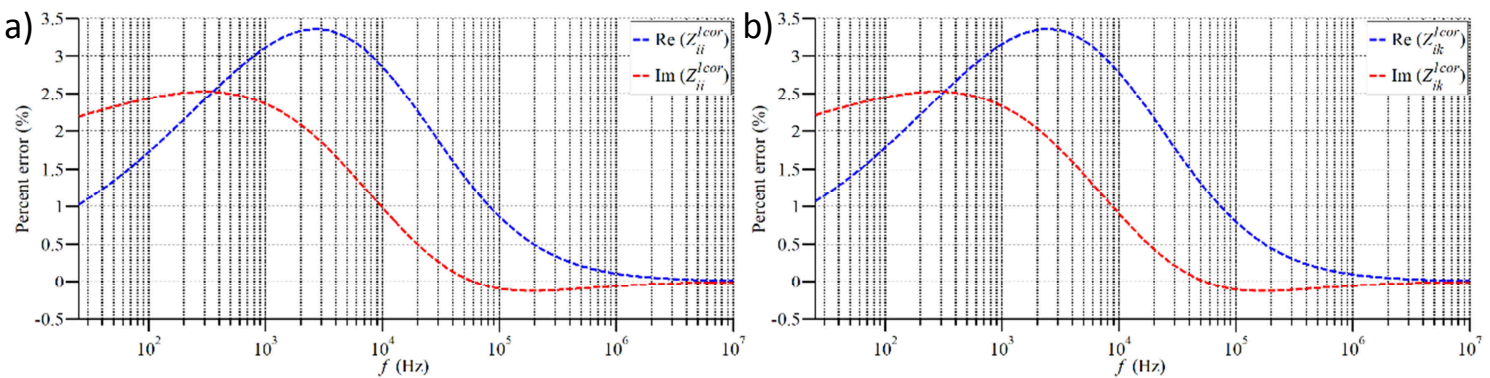

Fig. 7 Percent errors of Gary-Dubanton formulas for $\rho=10 \Omega m: a) \bar{Z}_{i i}^{1 c o r}$ and b) $\bar{Z}_{i k}^{1 \text { ik }}$ 

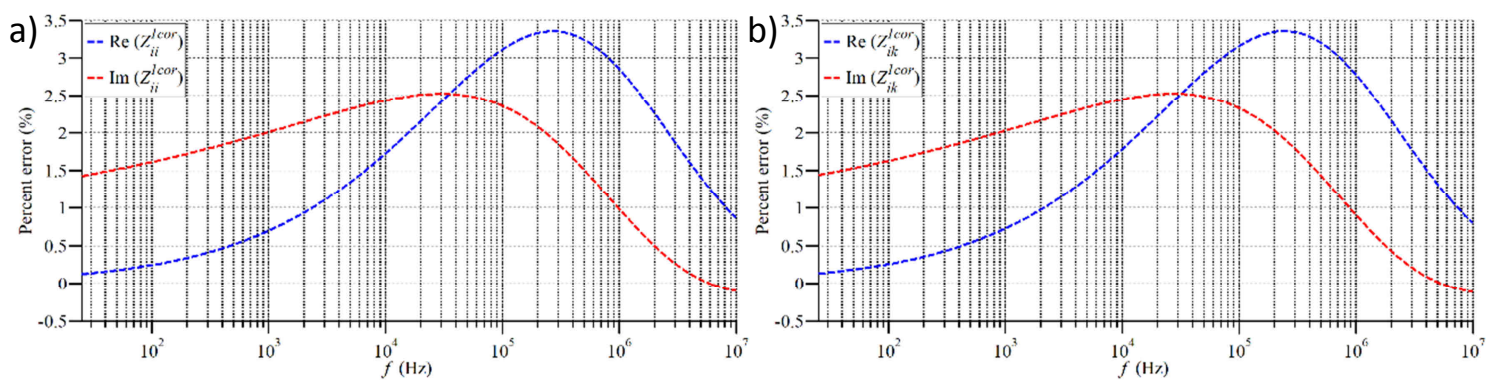

Fig. 8 Percent errors of Gary-Dubanton formulas for $\rho=1000 \Omega m: a) \bar{Z}_{i i}^{1 c o r}$ and b) $\bar{Z}_{i k}^{1 c o r}$
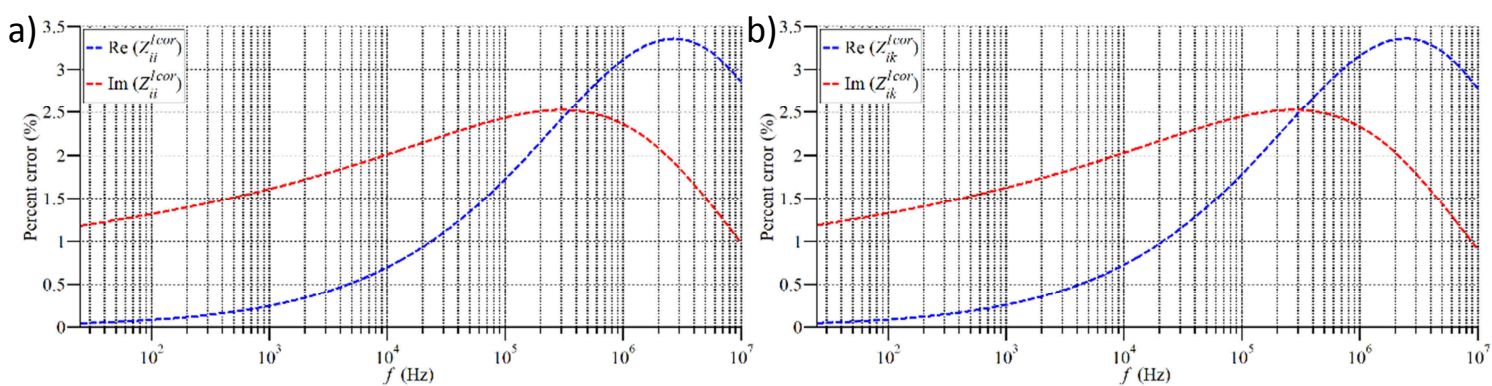

Fig. 9 Percent errors of Gary-Dubanton formulas for $\rho=10000 \Omega m:$ a) $\bar{Z}_{i i}^{1 \text { icor }}$ and b) $\bar{Z}_{i k}^{1 c o r}$

Percent errors of the Single-term approximation method are significantly higher at high frequencies, hence they are inappropriate for the graphic illustration. The maximum absolute and percent errors of both approximate formulas in relation to the 7-digit result for both pul impedance corrections are presented in Tables 2 to 4 .

Table 2 Maximum absolute and percent errors of both impedance corrections in relation to 7-digit accurate results $(\rho=10 \Omega m)$

\begin{tabular}{|c|c|c|c|}
\hline & Approximation method & Absolute error $(\Omega)$ & Percent error (\%) \\
\hline \multirow{2}{*}{ Q } & Gary-Dubanton formulas & $2.2377 \cdot 10^{-4}$ & 3.3570 \\
\hline & Single-term approximation & 9.5612 & $3.1006 \cdot 10^{3}$ \\
\hline \multirow{2}{*}{ 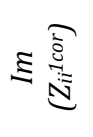 } & Gary-Dubanton formulas & $8.4694 \cdot 10^{-5}$ & 2.5241 \\
\hline & Single-term approximation & 43.2046 & $1.3667 \cdot 10^{4}$ \\
\hline \multirow{2}{*}{ 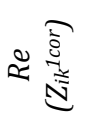 } & Gary-Dubanton formulas & $2.0184 \cdot 10^{-4}$ & 3.3596 \\
\hline & Single-term approximation & 9.5762 & $3.2638 \cdot 10^{3}$ \\
\hline \multirow{2}{*}{ 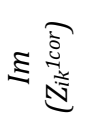 } & Gary-Dubanton formulas & $7.6462 \cdot 10^{-5}$ & 2.5249 \\
\hline & Single-term approximation & 43.8163 & $1.4586 \cdot 10^{4}$ \\
\hline
\end{tabular}


Table 3 Maximum absolute and percent errors of both impedance corrections in relation to 7-digit accurate results $(\rho=1000 \Omega m)$

\begin{tabular}{|c|c|c|c|}
\hline & Approximation method & Absolute error $(\Omega)$ & Percent error (\%) \\
\hline \multirow{2}{*}{ 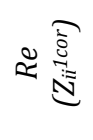 } & Gary-Dubanton formulas & $2.2377 \cdot 10^{-2}$ & 3.3570 \\
\hline & Single-term approximation & 7.3978 & 299.2854 \\
\hline \multirow{2}{*}{ 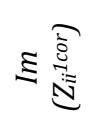 } & Gary-Dubanton formulas & $8.4694 \cdot 10^{-3}$ & 2.5241 \\
\hline & Single-term approximation & 17.0259 & 554.1361 \\
\hline \multirow{2}{*}{ 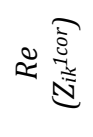 } & Gary-Dubanton formulas & $2.0184 \cdot 10^{-2}$ & 3.3596 \\
\hline & Single-term approximation & 7.4921 & 315.1237 \\
\hline \multirow{2}{*}{ 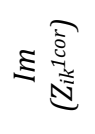 } & Gary-Dubanton formulas & $7.6462 \cdot 10^{-3}$ & 2.5249 \\
\hline & Single-term approximation & 17.5082 & 598.0664 \\
\hline
\end{tabular}

Table 4 Maximum absolute and percent errors of both impedance corrections in relation to 7-digit accurate results $(\rho=10000 \Omega m)$

\begin{tabular}{|c|c|c|c|}
\hline & Approximation method & Absolute error $(\Omega)$ & Percent error (\%) \\
\hline \multirow{2}{*}{ 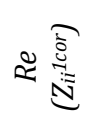 } & Gary-Dubanton formulas & 0.1455 & 3.3570 \\
\hline & Single-term approximation & 4.7733 & 93.6611 \\
\hline \multirow{2}{*}{ 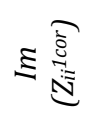 } & Gary-Dubanton formulas & $8.4694 \cdot 10^{-2}$ & 2.5241 \\
\hline & Single-term approximation & 7.8864 & 93.8790 \\
\hline \multirow{2}{*}{ 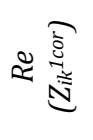 } & Gary-Dubanton formulas & 0.1383 & 3.3596 \\
\hline & Single-term approximation & 4.8955 & 98.4199 \\
\hline \multirow{2}{*}{ 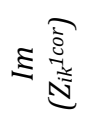 } & Gary-Dubanton formulas & $7.6462 \cdot 10^{-2}$ & 2.5249 \\
\hline & Single-term approximation & 8.1883 & 101.4012 \\
\hline
\end{tabular}

Results in Tables 3 to 5 show that the maximum percent errors of Gary-Dubanton formulas for pul self-impedance corrections are around 3,35\%, and the maximum percent errors for pul mutual impedance corrections are around 2,5\%, for all considered cases. Gary-Dubanton formulas have proven to be rather stable for different soil resistivities and at a larger frequency range. Single-term approximation method yields extremely unfavourable results at high frequencies (Figure 6). Results in Tables 3 to 5 show that the maximum percent errors of the Single-term approximation are significantly decreased for higher soil resistivities; however, these percent errors are still very high. The presented results confirm the statement that the Single-term approximation method is a rough approximation, valid only at low frequencies. 


\section{CONCLUSION}

High-accurate numerical algorithms for the computation of Carson formulas of the infinitely long parallel conductors are developed. The proposed algorithms are based on the piecewise linear and the piecewise quadratic approximation of the integrals' kernel function. The semiinfinite integrals for pul self and mutual impedance corrections are truncated by using the asymptotic approximation for large arguments. The kernel function of the integrals is identical in both cases, and it is independent of the frequency and soil characteristics. This fact was the key in developing a universal approximation algorithm valid at any frequency and for any soil characteristics. The approximated kernel function is multiplied by the rest of integrands and analytically integrated. By using both proposed numerical algorithms, the results are obtained with the desired computed n-digit accuracy easily. Piecewise linear algorithm has simpler expressions, whereas the piecewise quadratic algorithm has a significant reduction of total sample points. 7-digit accurate results obtained by the proposed algorithms were compared to the results obtained by the two approximation methods at a large frequency range. The comparison shows that the Single-term approximation method is valid at low frequencies and not appropriate at large frequencies. Gary-Dubanton formulas obtain reliable results throughout the entire frequency range considered in this paper.

\section{REFERENCES}

[1] J.R. Carson, Wave propagation in overhead wires with ground return, Bell System Technical Journal, Vol. 5, No. 4, pp. 539-554, 1926.

DOI: $10.1002 / j .1538-7305.1926 . t b 00122 . x$

[2] E.D. Sunde, Earth Conduction Effects in Transmission Systems, 2 ${ }^{\text {nd }}$ ed., New York: Dover, pp. 99-139, 1968.

[3] C. Dubanton, Calcul Approchè des Paramètres Primaires et Secondaires d'une Ligne de Transport. Valeurs Homopolaires, E.D.F. Bulletin de la Direction des Études et Recherches, Série B-Réseaux Électriques Matériels, No. 1, pp. 52-62, 1969.

[4] C. Gary, Approche complète de la propagation multifilaire en haute fréquence par utilisation des matrices complexes, E.D.F. Bulletin de la direction des Études et Recherches, Série B-Réseaux Électriques Matériels, No. 3/4, pp. 5-20, 1976.

[5] A. Deri, G. Tevan, A. Semlyen and A. Castanheira, The complex ground return plane a simplified model for homogeneous and multi-layer earth return, IEEE Transactions on Power Apparatus and Systems, Vol. PAS-100, No. 8, pp. 3686-3693, 1981.

DOI: $10.1109 /$ mper.1981.5511760

[6] Y.-J. Wang and S.-J. Liu, A review of method for calculation of frequency-dependent impedance of overhead power transmission lines, Proceedings of the National Science Council ROC (A), Vol. 25, No. 6, pp. 329-338, 2001.

[7] F.L. Alvarado and R. Betancourt, An Accurate Closed-Form Approximation for Ground Return Impedance Calculations, Proceedings of the IEEE, Vol. 71, No. 2, pp. 279-280, 1983. DOI: $10.1109 /$ proc. 1983.12573 
[8] O. Ramos-Leaños, J. L. Naredo, F. A. Uribe and J. L. Guardado, Accurate and Approximate Evaluation of Power-Line Earth Impedances Through the Carson Integral, IEEE Transactions on Electromagnetic Compatibility, Vol. 59, No. 5, pp. 1465-1473, 2017. DOI: $\underline{10.1109 / \text { temc.2017.2679213 }}$

[9] I. Krolo, T. Modrić and S. Vujević, Definition and computation of Carson formulas, $2^{\text {nd }}$ International Multidisciplinary Conference on Computer and Energy Science (SpliTech 2017), Split, pp. 1-6, 2017.

[10] I. Krolo, S. Vujević and T. Modrić, Computation of Carson formulas Using Piecewise Quadratic Approximation, 25 $5^{\text {th }}$ International Conference on Software, Telecommunications and Computer Networks (SoftCOM 2017), Split, pp. 1-7, 2017. DOI: $10.23919 /$ softcom.2017.8115518

[11] Central Station Engineers, Electrical Transmission and Distribution Reference Book, Westinghouse Electric Corporation, 4th ed., East Pittsburgh, 1964.

[12] W.H. Kersting, Distribution System Modeling and Analysis, $3^{\text {rd }}$ ed., Boca Raton: CRC Press, 2012. DOI: $\underline{10.1201 / \mathrm{b} 11697}$

[13] T. Gönen, Electrical Power Transmission System Engineering - Analysis and Design, $3^{\text {rd }}$ ed., Boca Raton: CRC Press, 2014. DOI: 10.1201/b17055

[14] A. Sommerfeld, Partial differential equations in physics, Academic Press, New York, 1949. DOI: $10.1016 / \mathrm{b} 978-0-12-654658-3 . \times 5001-0$

[15] S. Vujević, T. Modrić and B. Vukić, Internal impedance of two-layer cylindrical conductors, International Review of Electrical Engineering, Vol. 9, No. 1, pp. 235-243, 2014.

[16] S. Vujević, D. Lovrić and V. Boras, High-accurate numerical computation of internal impedance of cylindrical conductors for complex arguments of arbitrary magnitude, IEEE Transactions on Electromagnetic Compatibility, Vol. 56, No. 6, pp. 1431-1438, 2014. DOI: $\underline{10.1109 / \text { temc.2014.2352398 }}$

[17] D. Lovrić and S. Vujević, Accurate Computation of Internal Impedance of Two-Layer Cylindrical Conductors for Arguments of Arbitrary Magnitude, IEEE Transactions on Electromagnetic Compatibility, Vol. 60, No. 2, pp. 347-353, 2018.

DOI: $\underline{10.1109 / \text { temc.2017.2715985 }}$

[18] H. Cohen, Numerical Approximation Methods: $\pi \approx 355 / 113$, Springer, New York, 2011. DOI: $\underline{10.1007 / 978-1-4419-9837-8}$ 